\title{
Design of a Radiation Hardened Register File for Highly Reliable Microprocessors
}

\author{
Ramin Rajaei* \\ Department of Electrical Engineerimg, Shahid Beheshti Univeristy, Tehran, PO BOX: 1983963113, Iran
}

\begin{abstract}
In this paper, a powerful bit upset masking (PBUM) technique for design of a high reliable register file is proposed. This technique is based on the triple modular redundancy (TMR) technique with the key capability of double faulty bit masking in every triad of bits while the TMR structure, only masks one fault in a triad.

We implemented a 64-bit register file comprised of 64 registers protected with the proposed PBUM technique on FPGA. Our simulation results reveal that, over the TMR and some Hamming code-based techniques, our design offers a very higher robustness against radiation induced soft errors. Also, the proposed PBUM technique imposes a lower delay than its counterparts at the expense of a little higher area overhead. To reduce the area overhead, an area-efficient strategy is suggested that balances the reliability improvement and the area overhead. We show that, our technique using this area-aware strategy still has the highest reliability among the other considered techniques.
\end{abstract}

Index Terms: Triple Modular Redundancy (TMR), Single Event Upset (SEU), Multiple Bit Upset (MBU), Register File, Fault Tolerance.

(C) 2016 Published by MECS Publisher. Selection and/or peer review under responsibility of the Research Association of Modern Education and Computer Science.

\section{Introduction}

Radiation induced soft errors are of the serious concerns in design of highly reliable systems for the safety critical applications. High energy particles striking the sensitive nodes of an static random access memory (SRAM) cell, could cause a bit upset and result in operation failure [1-6]. The phenomenon of a bit flip in a memory cell such as a flip-flop or latch induced by a particle strike is called single-event upset (SEU). When an energetic particle hits more than one memory cell and causes more than one bit flip, a multi-bit upset (MBU) is happened [6-8].

On the one hand, the memory elements are widely used in microprocessor architectures with increasing trend in volume, and on the other, the technology dimension is shrinking to get higher compaction as well as speed

* Corresponding author. Tel.: +982122904105

E-mail address: r_rajaei@ sbu.ac.ir 
[5]. Technology shrinking that comes with decrease in supply voltage, affects the sensitivity of the SRAM cells to soft errors [5-8]. Fig.1 depicts the relation between the technology size, supply voltage and $\mathrm{Q}_{\text {crit }}$ (the minimum charge needed for a particle strike to result in an SEU). One can see that with decrease in the supply voltage and technology size, $\mathrm{Q}_{\text {cirt }}$ lowers more and more that is, the sensitivity to soft errors increases [9-10].

A number of techniques have been proposed to protect the SRAM based memory cells against SEUs. The most prominent and widely used technique, employable for reliability enhancement in flip-flops, level sensitive latches and SRAM memories is triple modular redundancy (TMR) [3, 9]. In this technique, each memory cell is triplicated and voted using a voter circuitry to mask one bit upset in one of the triplicated cells.

To protect the sequential logic, double modular redundancy (DMR) and TMR techniques are of the most effective gate-level techniques which can mask SEUs in Flip-flops. In [10], a comparison is made between the DMR (shown in Fig.2) and the TMR techniques.

To protect register files and cache memories in a microprocessor architecture, several parity-based techniques are proposed $[4,9,11]$. Comparing with the TMR technique, these methods impose less area overhead while, they usually cause more delay $[9,13]$.

In this paper, we suggest a new fault tolerant architecture for the register files. The proposed architecture is similar to the TMR register file (shown in Fig.3) with tripled voters differing in voting mechanism. To evaluate the reliability improvement of our proposed design and investigate its area and delay overhead, we implemented a register file composed of a sixty four 64-bit registers on an FPGA employing our technique in addition to some of previous techniques (see section 4). To slake the area overhead of our design, we also suggested to protect only the most accessible registers by our technique and protect the others with a less area consuming technique. The last sub-section of section 4, presents the trade-off between the area overhead and the reliability improvement offered by the proposed technique. In the next section of this paper, some related techniques for reliable design of register file are reviewed.

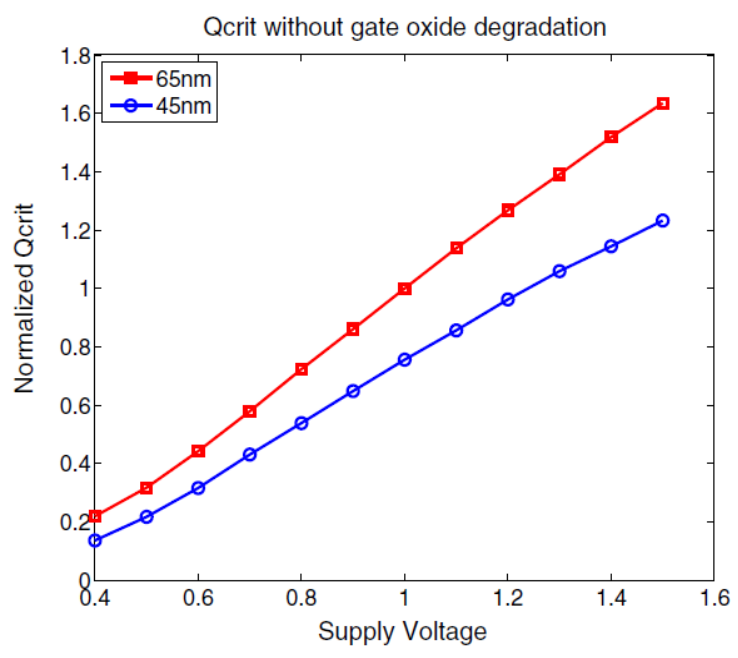

Fig.1. Scaling of Critical Charge for a SRAM Cell Respect to Voltage Scaling in 65nm and 45nm Technology Sizes [8]

\section{Related Work}

Several techniques based on Hamming code have been previously proposed to protect the register files against radiation induced soft errors. In [15], a single error correction/double error detection (SEC-DED) code is proposed. This code can detect and locate one bit error and detect double bit errors in a word. In [16-17], a code capable of detecting a byte error known as single error correction/double error detection/single byte error 
detection (SEC-DED-SBD) is proposed. These codes have more efficient performance than the conventional SEC-DED codes [1] at the expense of higher area overhead. In [2], four parity bits have been employed to correct up to four bit errors.

Another efficient fault tolerant technique for register files is using unused registers as copies of the used ones. This technique has been employed in superscalar processors [13]. In [4], duplication techniques, Hamming code $(\mathrm{H}(7,4))$ and narrow-width values are employed to enhance the reliability of the register files. Narrowwidth values are the values in which the upper half bits are identical. Therefore, they can be presented by halving the numbers of bits. As an example, suppose a 64-bit value has the upper 32-bits equal to 0, this value can be presented shorter using only 33 bits; The MSB can represent the value of the upper identical 32 bits and the remaining 32 bits for the lower 32 bits [4]. In [18], authors have proposed a new method for mitigating SEUs in register files. Their technique selectively copies the high vulnerable registers to soft error in a particular cache memory.

In [4] and [9], a useful analysis relevant to reliability of the TMR technique and some various Hamming codes is presented. The comparative analyses provided in section 4 employs this analysis provided in [4, 9]. A comprehensive analysis of sequential and combinational soft errors for the embedded processors is proposed in [19]. In [20], a quantification method for register vulnerabilities to soft error in register transfer logic (RTL) is proposed.

Another fault tolerant approach is radiation hardening by design. In this approach, radiation tolerance is obtained by a new design in circuit level. Radiation hardening by design can offer a high degree of reliability at the expense of a low design overhead [21-22]. However, they need specific fabrication process and high nonrecurring engineering (NRE) cost. Various circuits designed in [3, 5, 21, 23] offer SEU tolerance by design.

This paper proposes an SEU tolerant technique implementable in both the circuit and gate levels. The next section describes the idea behind the proposed technique.
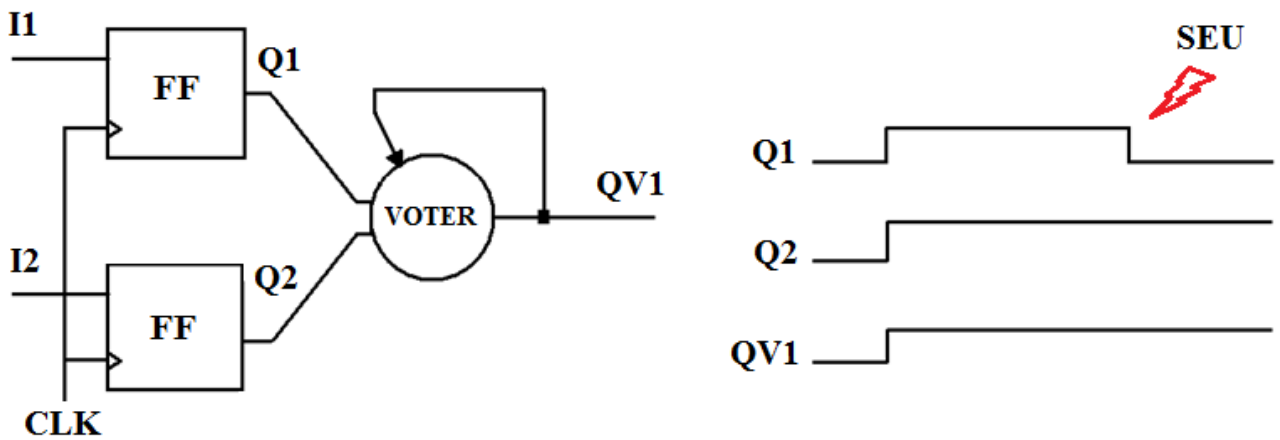

Fig.2. Structure Double Modular Redundancy with Feed-back Voter

\section{The proposed Powerful Bit Upset Masking (PBUM) Method}

Fig.3 shows a TMR register file. In this structure, every bit is triplicated and a voter circuit is used. This structure is capable of masking one bit upset in one of the triplicated bits. The proposed structure shown in Fig.4, has modified the voting structure so that, every two bits are voted using a three input voter for which the third input is a feedback from the output. In the presence of an SEU happening in one of the bits, the voter votes between the faulty bit, the correct bit and the previous correct state of the output. Therefore, the output will still remain correct as depicted in Fig.4 where every bit is showed by a flip-flop and the voting mechanism is shown by a feedback voter. In the structure shown in Fig.3, if an energetic particle affects two adjacent bits of a triad, only one of the voters will result a faulty output, while the other outputs will remain correct. Therefore, the last three-majority voter will result in a true logic value. In this structure, for every triad of bits, 
two faulty bits can be masked while in the similar TMR structure; only one faulty bit will be masked. This capability can be achieved in the 5MR structure where five bits using a 5-majority voter are employed imposing an extremely large area overhead.

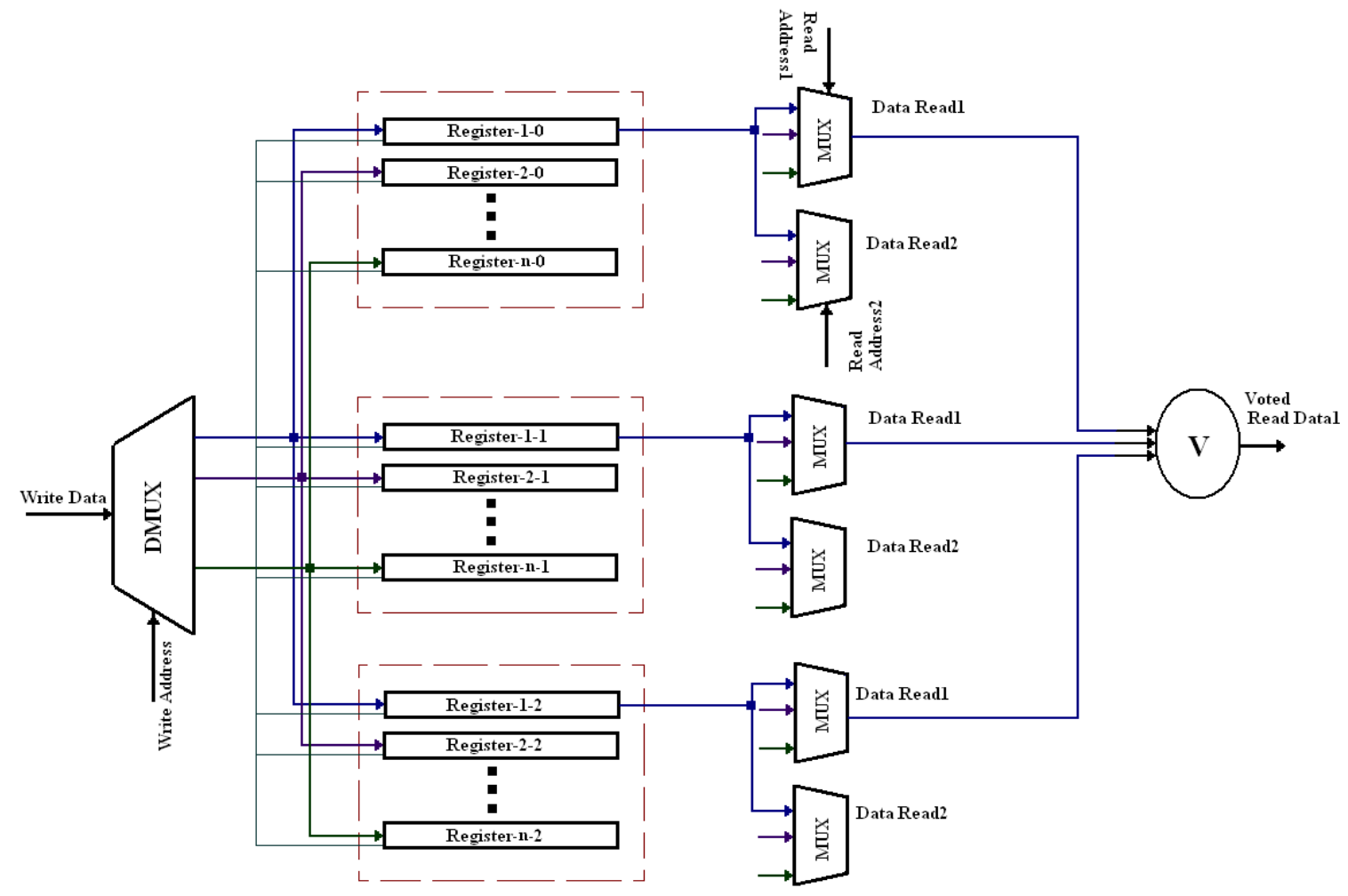

Fig.3. Structure of TMR Register File

\section{Comparative Analysis}

In this section, we analyze our design in terms of reliability, area and propagation delay and compare with some other previous techniques.

\subsection{Reliability Analysis}

Usually, there is a tradeoff between the reliability and the design overhead imposed for the reliability [3]. Developing a fault tolerant system with more reliability and less redundancy would be an asset. In [9], a mathematical analysis for investigating the probability of soft error affect in the TMR technique and the SECDEC codes is presented. In [4], a similar analysis for their proposed method is investigated and is compared with the TMR and Hamming code-based techniques. In evaluation of a fault tolerant technique, it is important to find out the quantity of reliability benefit offered by the technique.

According to [9], if the error probability in a single bit is $e$, the error probability of a word consisting of $w$-bit is given by:

$$
P_{\text {word-unprotected }}=1-(1-e)^{W}
$$


Applying the TMR technique, the error probability for the $w$-bit word can be found by [9]:

$$
P_{\text {Wrord-TMR }}=1-\left(1-3 e^{2}(1-e)-e^{\mathrm{a}}\right)^{\mathrm{W}}
$$

Similarly, applying the extended Hamming code of H(n, k), the error probability can be obtained using (3). In this equation, it is assumed that, the word with $w$-bit is divided into $k$-bit blocks, each one protected using ( $n$ $k$ ) parity bits [4]. The mechanism of the technique can be found in details in [4].

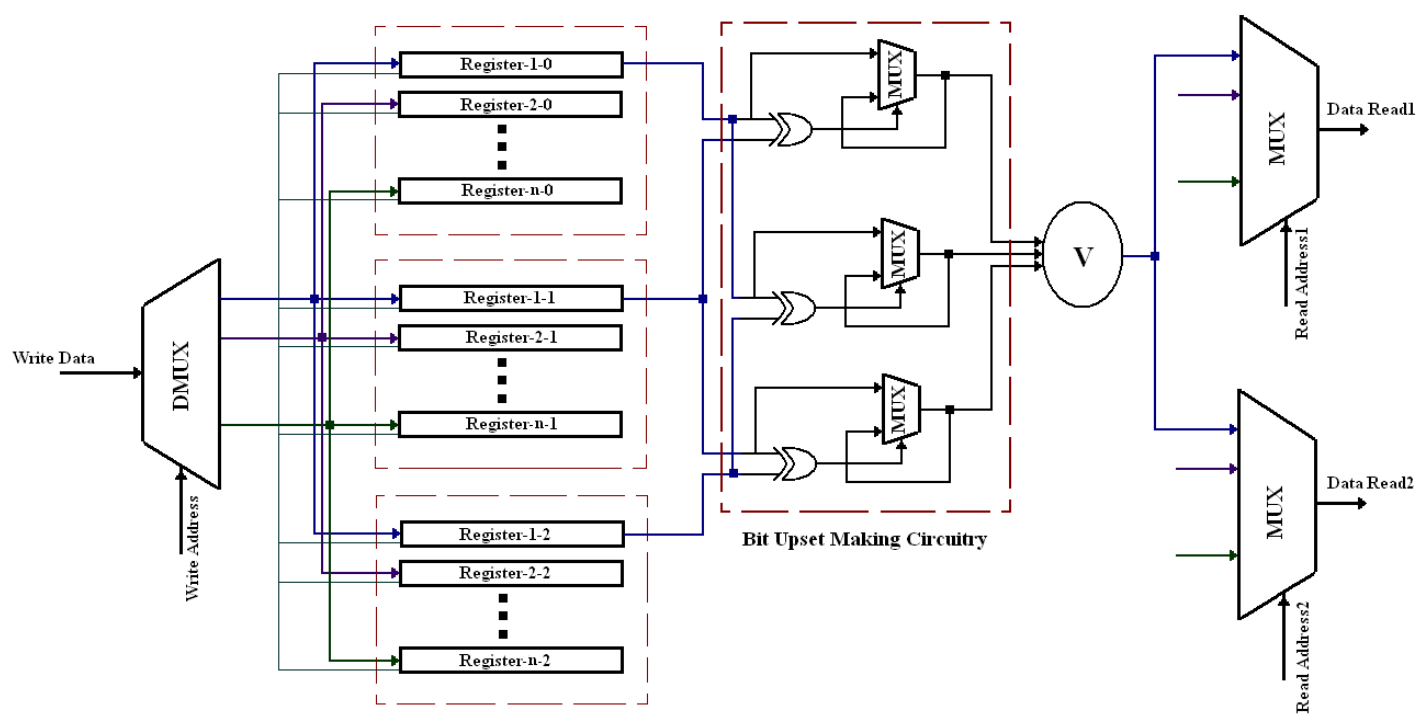

Fig.4. Structure of Proposed Register File

$$
P_{\text {word-extendedHamming }}=1-\left\{(1-e)^{n}+n e(1-e)^{(n-1)}\right\}^{\left(\frac{w}{k}\right)}
$$

In [4], the authors reported that, the error probability of their proposed method is computed using (4).

$$
P_{\text {word-proposed method in [6] }}=\text { pct }_{\text {normal }} \times P_{\text {word-normal }}+\text { pct } t_{\text {narrow }} \times P_{\text {word-narrow }}
$$

In which:

$$
\sum_{j=1}^{z=1}\left(1-c_{j}\right)\left(\frac{(2 w) n}{(2 w-j) ! \times j n}\right) e^{i}(1-e)^{[2 w-j]}
$$

Where, $C_{j}$ is correction percentage for $\mathrm{j}$ random errors in normal width values and:

$$
P_{\text {word-narrow }}=\sum_{j=1}^{2 w}\left(1-C_{j}\right)\left(\frac{(2 w) !}{(2 w-j) ! \times j !}\right) e^{j}(1-e)^{(2 w-j)}
$$

Where, $C_{j}$ in above relation is correction percentage for $j$ random errors in narrow width values [4]. As their 
simulation results reveal, $p c t_{\text {normal }}$ and $p c t_{\text {narrow }}$ for the SPEC2000 benchmark is $15 \%$ and $85 \%$ respectively. Similar to relations 1 to 4 , we can derive the error probability for the DMR (shown in Fig.2) and 5MR techniques. The relevant equations are:

$$
P_{\text {Word-DMR }}=1-\left(1-e^{2}\right)^{W}
$$

And:

$$
P_{\text {Word-5MR }}=1-\left(1-10 e^{a}(1-e)^{2}-5 e^{4}(1-e)-e^{5}\right)^{\text {W }}
$$

To calculate the error probability of our proposed circuit, it is considerable that our structure can mask 1 and 2-bit errors while the TMR can mask only one error. Therefore, the equation for error probability of a $w$-bit word is obtained by (7).

$$
P_{\text {word }- \text { our vrovosed method }}=1-\left(1-e^{\mathrm{a}}\right)^{\mathrm{w}}
$$

Fig.5, compares the error probability of the error correcting codes specified by equations 1 to 7 assuming a $w$ of 64 . As can be seen in this figure, the proposed technique could significantly improve the circuit reliability.

\subsection{Area and Delay overhead Investigation}

As mentioned earlier, the proposed PBUM technique for fault tolerant design of register files can be implemented in both the ASIC and FPGA designs. To investigate the area and delay overhead of PBUM, we employed it in design of a 64-bit register file comprised of 64 registers using the Verilog describing code and implemented it on a XC4VFX100 Xilinx FPGA. Some other approaches including the TMR register file and the protected ones by Hamming codes $(\mathrm{H}(7,4), \mathrm{H}(12,8), \mathrm{H}(21,16)$ and $\mathrm{H}(38,32))$ as well as the not protected register file are all implemented and compared with our proposed fault tolerant register file (shown in Fig.4).

To have a better comparison for the area consumed by the implemented techniques, we enforced the synthesizer to use flip-flop for implementation the register files. In Fig.6, the total combinational delay of the register files (not protected as well as the protected ones) are compared. As can be found from the results, our proposed structure and the TMR impose less delay overhead than the other fault tolerant structures.

Another cost is paid for the reliability is the consumed area. Usually highly reliable techniques impose large area penalty. Fig.7 compares the area overhead of the implemented techniques. As aforementioned, to have a fair comparison, we enforced the ISE to synthesize all the register file structures using flip-flops. The figure shows the used area in terms of the consumed FPGA slice, look-up table (LUT) and flip-flops. As was expected, the TMR and our proposed technique, impose more area overhead in comparison with the other fault tolerant register files while as Fig.5 shows, the reliability improvement of our proposed fault tolerant register file is significantly more than the TMR and also the other structures. In other words, our proposed register file is the best in terms of reliability and delay among the considered counterparts while it occupies more area. The next sub-section discusses on how we can lower the occupied area by our design keeping the reliability improvement the highest. 


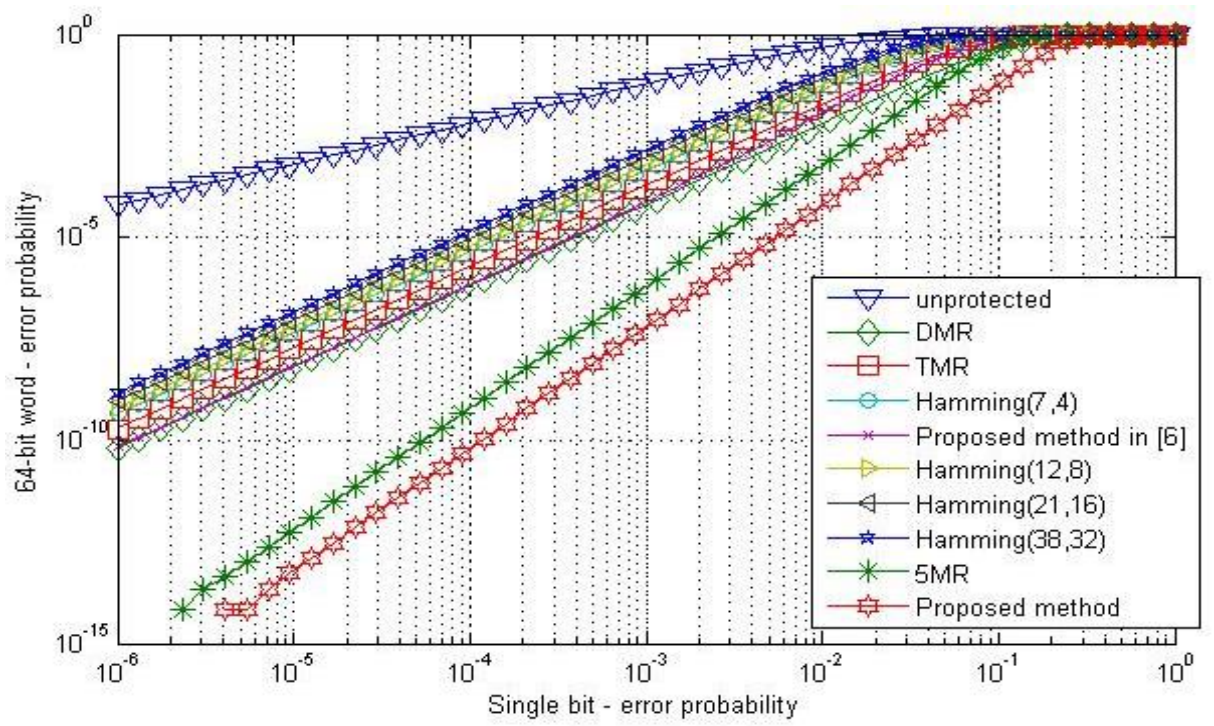

Fig.5. Error Probability Comparison of Various Techniques for a 64-bit Word Register File

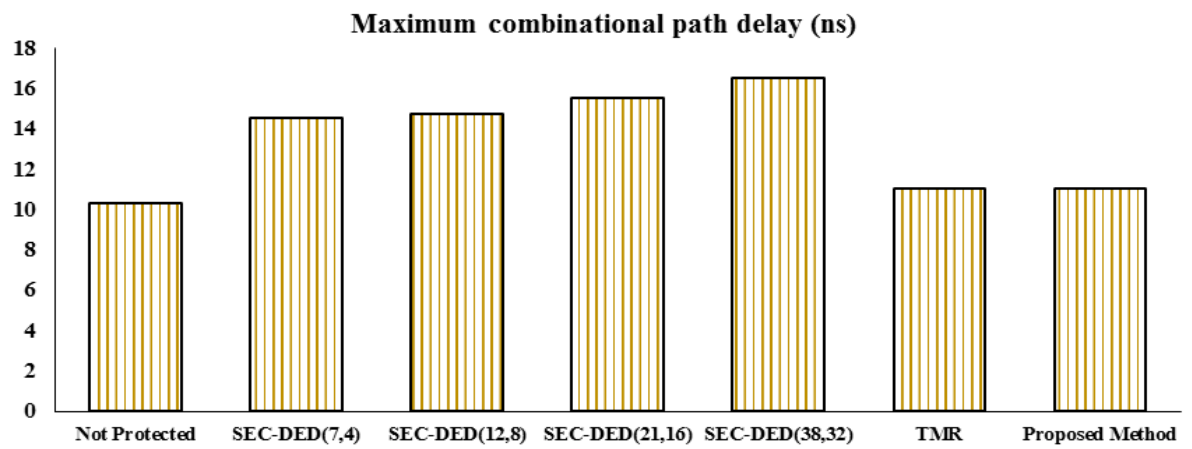

Fig.6. Comparison of Maximum Combinational Path Delay Made for Implemented 64-bit Word Register Files Protected by Various Techniques on XC4VFX100

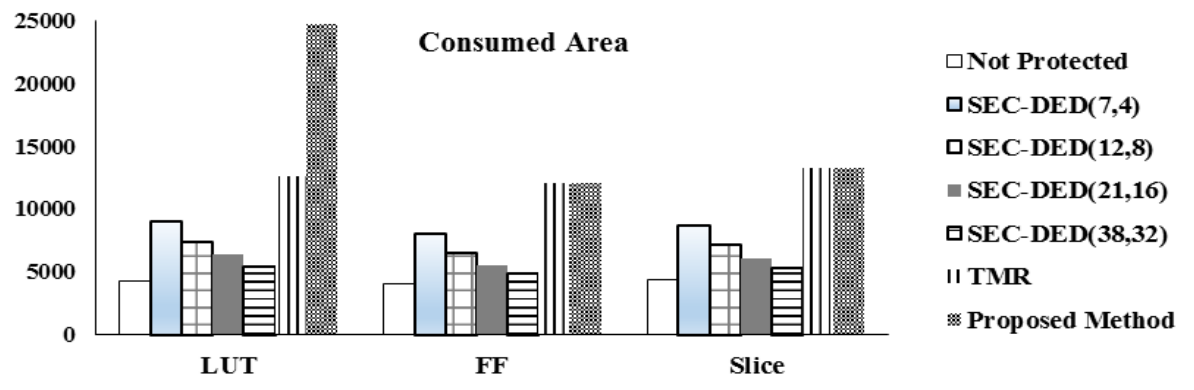

Fig.7. Comparison of used area in XC4VFX100 Xilinx FPGA. 


\subsection{Tradeoff between Area and Reliability}

In section 3, we protected all the bits of register file by the introduced PBUM technique. Doing this, we achieved a very reliable register file while it expenses area overhead. Our idea to slake the area overhead of the proposed technique, is efficiently applying the PBUM for a subset of registers instead of all. It is showed that, running a program on a processor, all the registers of register file are not being accessed equally [4, 18]. As a case study, we used MiBench [24] running on a 32-bit ARM microprocessor. According to the simulation results shown in Fig.8, on average, about $96.2 \%$ of register accesses are to registers 0 to 15, i.e. the others ones (registers 16 to 32 ) are accessed less than $4 \%$. It means that we can protect only one half of the register file using the proposed PBUM technique while the other half with less access can left un-protected or protected using a less area consuming techniques. It can significantly decrease the area overhead while the reliability will not be affected seriously.

In Fig. 9 and Fig.10, a comparison is made for normalized area overhead and reliability achievement of the TMR, our proposed technique, half protected using our technique and half protected using Hamming $(7,4)$, Hamming $(21,16)$ and DMR. As can be seen from the results presented in these figures, depending on the application a desirable trade-off for reliability and area can be found.

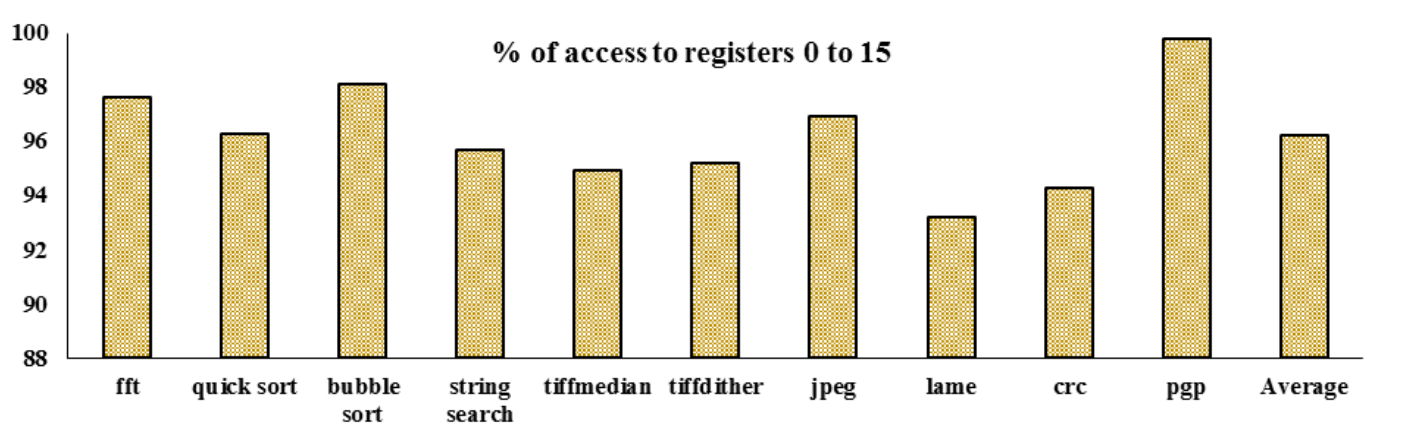

Fig.8. Percentages of access to Registers 0 to 15 in Various Benchmarks

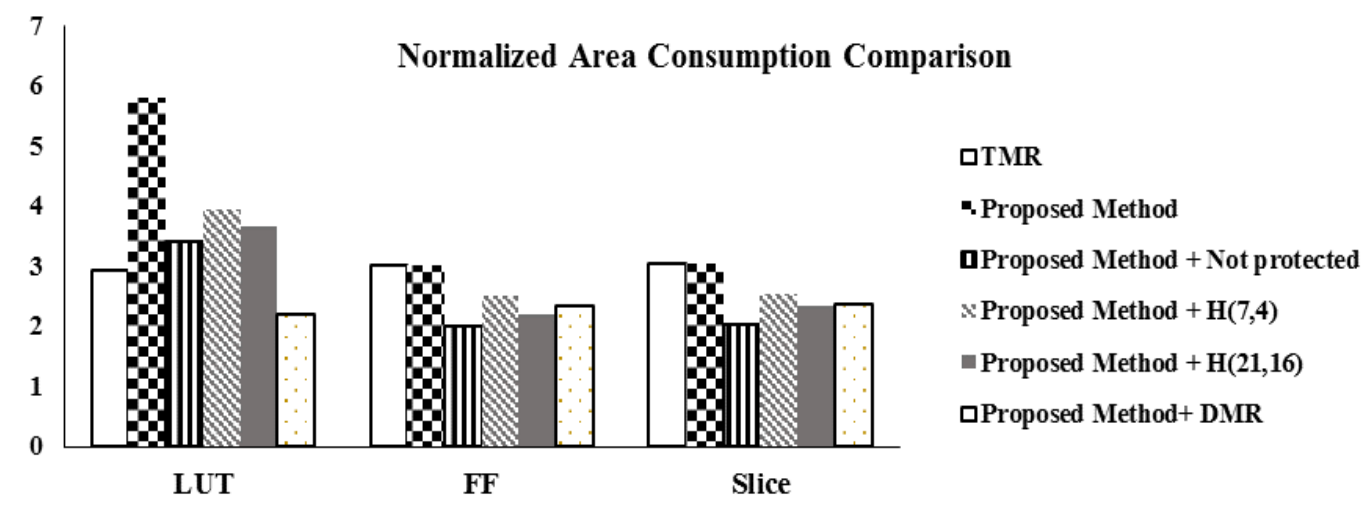

Fig.9. Comparison of Occupied Area in Various Techniques used the Suggested Strategy for Slake the area Overhead 


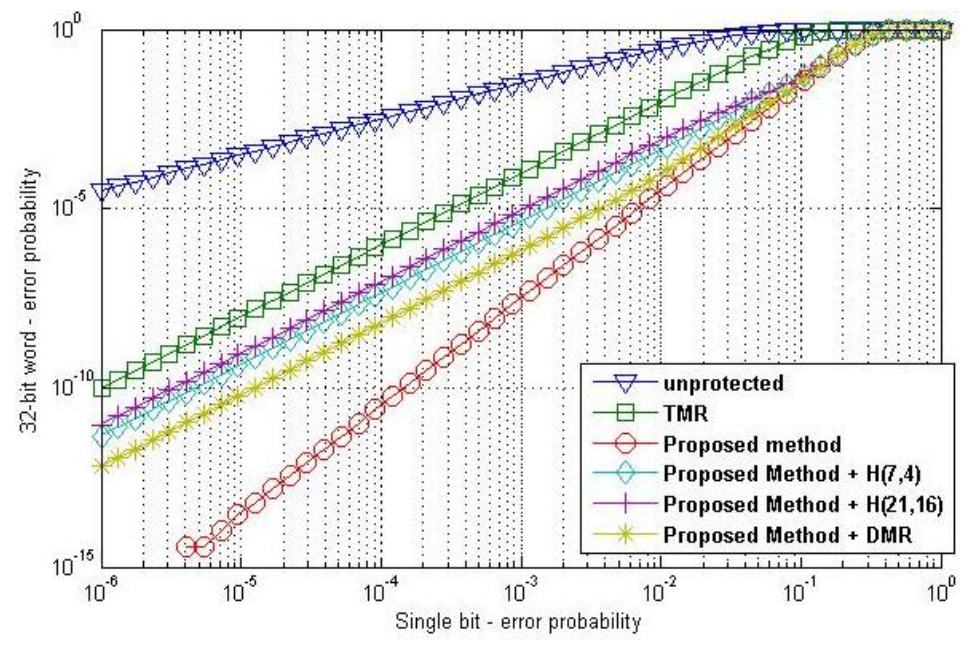

Fig.10. Comparison of Error Probability in Various Techniques used the Suggested Strategy for Slake the area Overhead

\section{Conclusion}

In this paper, we proposed a highly reliable register file for embedded processors. The proposed idea can be implemented in both the ASIC and FPGA designs. The proposed fault tolerant register file as well as some other fault tolerant register files are implemented on FPGA. The presented reliability analyses and area/performance evaluations of the designs showed that the proposed architecture decreases significantly the error probability and imposes a very small delay overhead. However, the proposed PBUM technique occupies larger area in comparison with the other considered techniques. To deal with this drawback, we suggested an area efficient strategy to design a reliable and low area register file. The proposed area aware strategy relays on an observation that the register access is not equal for all the registers, i.e. some registers usually being accessed more frequently than the others. This area overhead solution applies the proposed PBUM technique for the high accessed registers and uses another less area consuming technique for the registers with lower accessing rates. Applying this solution, the area overhead decreased significantly while the reliability remained the best among the other considered techniques.

\section{References}

[1] A. Dutta, N. A. Touba, "Multiple Bit Upset Tolerant Memory Using a Selective Cycle Avoidance Based SEC-DED-DAEC Code," In 25th IEEE VLSI Test Symmposium (VTS’07) (2007).

[2] A. J. Ricketts, M. Mutyam, N. Vijaykrishnan, N. J. Irwin, "Investigating Simple Low Latency Reliable Multiported Register Files," VLSI, 2007. ISVLSI '07. IEEE Computer Society Annual Symposium, vol., no., pp.375-382, 9-11 March 2007.

[3] R. Rajaei, M. Tabandeh, B. Rashidian, "Single Event Upset immune latch circuit design using CElement," in Proc. of the IEEE 9th International Conference on ASIC, China, pp. 280-283, 2011.

[4] S. Esmaeeli, M. Hosseini, B. V. Vahdat, B. Rashidian, "A multi-bit error tolerant register file for a high reliable embedded processor," 18th IEEE International Conference on Electronics, Circuits and Systems (ICECS), 2011.

[5] R. Rajaei, B. Asgari, M. Tabandeh, M. Fazeli, "Design of Robust SRAM Cells Against Single Event Multiple Effects for Nanometer Technologies," IEEE Transactions on Device and Materials Reliability, 2015. 
[6] B. Alidoosti, M. H. Moaiyeri, "An Energy-Efficient and Robust Voltage Level Converter for Nanoelectronics," International Journal of Modern Education and Computer Science (IJMECS), 7(5), 2015.

[7] R. Rajaei, B. Asgari, M. Tabandeh, M. Fazeli, "Single Event Multiple Upset-Tolerant SRAM Cell Designs for Nano-scale CMOS Technology," Turkish Journal of Electrical Engineering \& Computer Sciences, 2016.

[8] M. Kaviani, H. Sharifi, M. Dolatshahi, K. Navi, "Design of Low Voltage and High-Speed BiCMOS Buffer for Driving Large Load Capacitor," International Journal of Engineering and Manufacturing (IJEM), 2016.

[9] R. Naseer, R. Z. Bhatti, J. Draper, "Analysis of Soft Error Mitigation Techniques for Register Files in IBM Cu-08 90nm Technology," Circuits and Systems, MWSCAS '06. 49th IEEE International Midwest Symposium on , vol.1, no., pp.515-519, 6-9 Aug. 2006.

[10] V. Chandra, R. Aitken, "Impact of voltage scaling on nanoscale SRAM reliability," Design, Automation \& Test in Europe Conference \& Exhibition, 2009. DATE '09.

[11] R. Rajaei, M. Tabandeh, M. Fazeli, "Low Cost Soft Error Hardened Latch Designs for Nano-scale CMOS Technology in presence of Process Variation," Microelectronic Reliability, Elsevier, 2013.

[12] R. Gong, W. Chen, F. Liu, K. Dai, Z. Wang, "Modified Triple Modular Redundancy Structure based on Asynchronous Circuit Technique," in 21th IEEE International Symposium on Defect and Fault-Tolerance in VLSI Systems (DFT 2006), Arlington, Virginia, USA. Pages 184-196, IEEE Computer Society, 2006.

[13] G. Memik, M.T. Kandemir, and O. Ozturk "Increasing Register File Immunity to Transient Errors", Proceedings of Design, Automation and Test in Europe (DATE’05), Munich, Germany, vol. 1, pp. 586591, Mar. 2005.

[14] R. Hentschke, F. Marques, F. Lima, L. Carro, A. Susin, R. Reis, "Analyzing Area and Performance Penalty of Protecting Different Digital Modules with Hamming Code and Triple Modular Redundancy". Proceedings of the 15 th Symposium on Integrated Circuits and Systems Design (SBCCI'02).

[15] R. W. Hamming, "Error Correcting and Error Detecting Codes", Bell Sys. Tech. Journal, Vol. 29, pp. 147-160, Apr. 1950.

[16] S. M. Reddy, "A Class of Linear Codes for Error Control in Byte-per-Package Organized Memory Systems”, IEEE Trans. On Computers, Vol. C-27, pp. 455-458, May. 1978.

[17] C. L. Chen, "Error Correcting Codes with Byte Error Detection Capability", IEEE Trans. On Computers, Vol. C-32, pp. 615-621, May 1983.

[18] M. Fazeli, S. A. Ahmadian, S.G. Miremadi, “A Low Energy Soft Error-Tolerant Architecture for Register File in Embedded Processors, 11th IEEE High Assurance Systems Engineering Symposium, (HASE 2008), Nanjing, China, 2008.

[19] M. Ebrahimi, A. Evans, M.B. Tahoori, E. Costenaro, D. Alexandrescu, V. Chandra, R. Seyyedi, "Comprehensive Analysis of Sequential and Combinational Soft Errors in an Embedded Processor," in IEEE Transactions on Computer-Aided Design of Integrated Circuits and Systems (TCAD), 2015.

[20] L. Chen, M. Ebrahimi, M.B. Tahoori, "Formal Quantification of the Register Vulnerabilities to Soft Error in RTL Control Paths," Journal of Electronic Testing: Theory and Applications (JETTA), Springer, 2015.

[21] R. Rajaei, S. Bakhtavari, F. Eslaminasab, Radiation Hardening by Design for Nonvolatile Magnetic FlipFlops, The $1^{\text {st }}$ International Conference on New Research Achievements in Electrical and Computer Engineering, Tehran, Iran, 2016.

[22] B. Alidoosti, M. H. Moaiyeri, "An Energy-Efficient and Robust Voltage Level Converter for Nanoelectronics," International Journal of Modern Education and Computer Science (IJMECS), 7(5), 2015.

[23] R. Rajaei, "Radiation Hardened Design of Nonvolatile MRAM-based FPGA," IEEE Transactions on Magnetics, 2016.

[24] M. R. Guthaus, J. S. Ringenberg, D. Ernst, T. M. Austin, T. Mudge, and R. B. Brown, "MiBench: A free, commercially representative embedded benchmark suite," in Proc. IEEE 4th Annu. Workshop Workload 
Characterization, Austin, TX, Dec. 2001, pp. 3-14.

\section{Authors' Profiles}

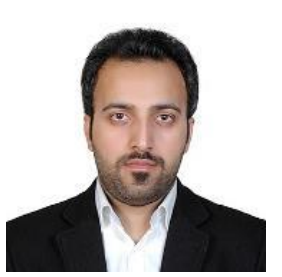

Ramin Rajaei received the M.Sc. and Ph.D. degrees in electrical engineering from the Sharif University of Technology (SUT), Tehran, Iran, in 2009 and 2014 respectively. He has been an Assistant Professor with the Department of Electrical Engineering, Shahid Beheshti University (SBU), Tehran, Iran, since 2015. His current research interests include reliability and power issues in ASIC/FPGA designs based on CMOS and also emerging technologies.

How to cite this paper: Ramin Rajaei,"Design of a Radiation Hardened Register File for Highly Reliable Microprocessors", International Journal of Engineering and Manufacturing(IJEM), Vol.6, No.5, pp.11-21, 2016.DOI: 10.5815/ijem.2016.05.02 